Revue

Revue de l'histoire des religions

del'histoire des religions

Irene BUENo et Camille ROUXPETEL (dir.), Les récits

historiques entre Orient et Occident ( $\mathrm{XI}^{e}-\mathrm{XV}^{e}$ siècle)

Rome, École française de Rome, 2019

Florian Besson

\title{
OpenEdition
}

Journals

Édition électronique

URL : http://journals.openedition.org/rhr/11064

DOI : 10.4000/rhr.11064

ISSN : 2105-2573

Éditeur

Armand Colin

Édition imprimée

Date de publication : 1 mars 2021

Pagination : 151-154

ISBN : 978-2-200-93375-3

ISSN : 0035-1423

Référence électronique

Florian Besson, «Irene Bueno et Camille Rouxpetel (dir.), Les récits historiques entre Orient et Occident ( $x l^{e}-x v^{e}$ siècle) », Revue de l'histoire des religions [En ligne], 1| 2021, mis en ligne le 19 mars 2021, consulté le 31 mars 2021. URL : http://journals.openedition.org/rhr/11064 ; DOI : https://doi.org/ 10.4000/rhr.11064

Ce document a été généré automatiquement le 31 mars 2021.

Tous droits réservés 


\section{Irene BUENO et Camille ROUXPETEL (dir.), Les récits historiques entre Orient et Occident ( $\mathrm{XI}^{e}-\mathrm{XV}^{e}$ siècle)}

Rome, École française de Rome, 2019

\section{Florian Besson}

\section{RÉFÉRENCE}

Irene BUENo et Camille ROUXPETEL (dir.), Les récits historiques entre Orient et Occident (XI ${ }^{e}$ $X V^{e}$ siècle), Rome, École française de Rome, 2019, 384 p., 24 cm, $41 €$, ISBN

978-2-7283-1362-4.

1 Les dix contributions réunies dans cet ouvrage dirigé par Irene Bueno et Camille Rouxpetel s'attachent à étudier la circulation des "récits historiques", autrement dit des textes racontant l'histoire, entre l'Occident chrétien et l'Orient ; ou plutôt, faudraitil dire d'emblée, entre les Orients, tant sont divers les univers - religieux, ecclésiologiques, politiques, linguistiques - qui sont ici explorés et interrogés. Précisons d'ailleurs d'emblée que ces Orients sont essentiellement des Orients chrétiens: si certains auteurs font occasionnellement référence à des sources islamiques, la focale est davantage centrée sur la question des relations entre chrétienté latine et chrétientés d'Orient, en particulier l'Église arménienne, à laquelle quatre articles sont consacrés. Il s'agit bien plus ici d'un choix méthodologique, afin de donner une cohérence à l'ouvrage, que d'un oubli: la conclusion rappelle d'ailleurs la nécessité d'élargir cette enquête dans l'avenir, pour interroger également l'écriture de l'histoire dans le monde islamique médiéval.

Comme le rappellent les deux directrices d'ouvrage dans leur excellente introduction, les récits historiques ont été très étudiés par les médiévistes, qui se sont attachés à montrer les complexes rapports qu'ils nouent avec le passé, mais aussi avec le présent. Par rapport à cette riche historiographie, construite notamment autour des travaux de 
Bernard Guenée, le présent ouvrage fait un pas de côté : il s'agit en effet de se concentrer sur les circulations "horizontales» de textes, entre les deux rives de la Méditerranée. En s'intéressant aux échanges de textes, il s'agit évidemment de mieux comprendre la manière dont des sociétés à la fois très différentes et très proches entrent en contact, échangent, apprennent les unes des autres. L'actualité historiographique de ces questions, qui recoupe par exemple la question d'histoire médiévale inscrite au programme de l'agrégation interne d'histoire en 2018-2020, est aussi évidente que l'est leur actualité sociale. Le sujet est ambitieux et le cadre est très large, tant chronologiquement - cinq siècles, mais en réalité certains articles remontent jusqu'au $v^{\mathrm{e}}$ siècle de notre ère - que géographiquement, puisque l'on va de Rome à l'Éthiopie, de l'Asie Centrale au Portugal, de l'Allemagne au Yémen. Après un premier article plus théorique, dans lequel Frédéric Gabriel revient sur l'élaboration exégétique d'un texte biblique à même de se faire matrice d'identités sans cesse en évolution, les différentes contributions se penchent sur des exemples très variés. De la transmission de l'histoire éthiopienne ou arménienne à la Curie romaine $\mathrm{du} \mathrm{xv}^{\mathrm{e}}$ siècle jusqu'aux tentatives - laborieuses - de rapprochement entre les Églises grecque et arménienne au $\mathrm{XII}^{\mathrm{e}}$ siècle, en passant par l'utilisation par la papauté de la Donation de Constantin, le livre embrasse des espaces, des acteurs et des langues très diverses. Plutôt que de mentionner successivement tous les articles du livre, l'on dégagera ici trois lignes de force qui les traversent et les articulent.

3 L'un des grands intérêts du livre est d'abord de bien faire voir, dans tous les articles, que la circulation très concrète des textes est toujours sous-tendue par des circulations moins visibles : circulations d'hommes, d'idées, de récits oraux, etc. À bien des égards, les récits historiques apparaissent comme la pointe d'un iceberg immense, mais largement invisible pour les historiens contemporains : derrière l'histoire de la culture matérielle se devine, plus ou moins effacée, toute une histoire intellectuelle. Les différentes méthodes employées par les auteurs des articles - qu'il s'agisse de reconstituer patiemment la généalogie d'une anecdote, de s'intéresser aux variations dans les copies d'un même texte ou encore d'étudier la chaîne de transmission d'un récit - servent ainsi à mettre en valeur le dynamisme d'un monde médiéval bien plus densément connecté qu'on ne l'a longtemps pensé.

4 Le deuxième intérêt du livre est de rappeler en permanence que derrière ces circulations se dissimulent toujours des objectifs, et les différentes contributions rassemblées ici se focalisent notamment sur la question des stratégies ecclésiologiques : quand le pape écrit au Negus éthiopien en lui racontant l'histoire de la conquête éthiopienne du royaume d'Himyar, objet de l'article de Benjamin Weber, il s'agit moins de nouer un lien de connivence que de rappeler l'antique soumission des Éthiopiens au basileus byzantin, désormais transférée sur le pontife romain... Derrière le récit historique se dissimule donc en l'occurrence un objectif politique visant, à l'heure du concile de Florence, à affirmer la suprématie de la papauté romaine et à faire du pape la clé de voûte d'un programme de réunification du monde chrétien. C'est en vue d'un objectif très proche que la papauté s'intéresse à l'histoire arménienne, comme le montre Irene Bueno dans son texte: en rappelant ou, le cas échéant, en réécrivant l'histoire des premiers conciles, il s'agit avant tout de légitimer l'alliance entre Latins et Arméniens, et de s'en servir pour revivifier un idéal de croisade un peu terni. Quant à la figure du "grand Khan" prêt à se convertir au christianisme, qu'étudie Thomas Tanase, elle devient, au fil de ses réécritures successives en Occident, l'une des 
fondations des projets d'exploration atlantique portés par le Portugal à la fin du $\mathrm{XV}^{\mathrm{e}}$ siècle. À l'inverse, certains textes semblent largement imperméables aux urgences de leur époque : comme le montre ainsi Marco Biais, l'historien arménien T'ovma Mecop'ec'i ne mentionne pas dans son Histoire de Tamerlan les complexes débats et rivalités entre Églises orientales, alors même qu'il y était par ailleurs engagé.

Enfin, un dernier point fort de l'ouvrage est l'attention portée, là encore dès l'introduction, aux ambivalences de ces circulations. Un texte, en effet, ne voyage jamais "par hasard», pas plus qu'il ne voyage sans être transformé, parfois superficiellement - ainsi le nom propre d'un souverain sera-t-il modifié au fil des traductions successives d'un récit en grec, en arabe, en guèze et en latin - parfois structurellement. Au fil des articles se dégage une véritable typologie de ces modifications, que la conclusion, peu utile en l'état, aurait pu tenter de rappeler. Si les manipulations volontaires sont finalement plus rares qu'on pourrait a priori le penser, l'éventail reste assez large : processus de "dématérialisation historique ", tel celui qui fait du Kubylay Khan de Marco Polo, acteur historique ayant vraiment existé, un «Grand Khan » fantomatique et éternel; exagération croissante au fil des réécritures d'un même événement ou élément ; confusion, volontaire ou non, amenant à fusionner deux acteurs distincts ou à mélanger deux conciles; stratégie rhétorique insistant sur une lecture prophétique et eschatologique de l'histoire, en profitant de l'avantage qu'offre le fait d'écrire après les événements; et bien d'autres encore. Ces modifications en disent long sur les contextes de réception, et donc in fine sur les cultures respectives des sociétés qui reçoivent ainsi ces récits historiques venus d'ailleurs, les réinterprètent et ce faisant se les réapproprient. Les déformations, les malentendus, les incompréhensions qui émaillent cette histoire ne sont donc pas des scories venant polluer la «bonne » transmission de récits, mais participent pleinement d'une histoire riche et complexe des échanges culturels en Méditerranée médiévale. Dans son article, Thomas Tanase invite ainsi à ne pas considérer les textes historiques uniquement comme des sommes de connaissances qui seraient progressivement dépassées par la découverte de nouveaux éléments et par le flux permanent des événements: la description par Marco Polo d'un grand Khan favorable au message chrétien façonne au contraire les imaginaires géographiques, politiques et ecclésiologiques sur le long terme, au point de devenir une véritable « couche géologique » qui sous-tend encore aujourd'hui en partie notre vision du monde. La frontière entre "réception" et «création» devient alors extrêmement floue. Yuri P. Avvakumov montre ainsi que l'Anticimenon d'Anselme d'Haverberg ne renvoie pas à un véritable débat entre Grecs et Latins : le texte n'est qu'une fiction, mais une fiction qui permet, précisément, d'étudier la façon dont un évêque allemand pense l'unité de la foi chrétienne en dépit, ou à travers, l'extrême diversité géographique de ses rites.

6 La densité de l'ouvrage est à la hauteur de la grande érudition de ses auteurs. Si on regrettera occasionnellement que certains articles ne soient pas davantage reliés au thème central - c'est en particulier le cas pour la très longue contribution de Dan Ioan Muresan sur la Donation de Constantin - reste que le livre a une grande cohérence. La question de la circulation des récits historiques entre Orient et Occident apparait comme une clé d'entrée particulièrement féconde, reposant à la fois sur des exemples précis et sur des concepts finement problématisés, pour penser les rapports culturels, politiques et ecclésiologiques entre les différentes sociétés de la Méditerranée 
médiévale. L'enquête demande assurément à être poursuivie et ouverte à de nouveaux terrains : l'histoire ne fait que commencer ! 